\title{
Clinical Analysis of the Effect of Contrast-enhanced UI- trasonography on Trans-arterial Chemoembolization for Advanced Liver Cancer in the Elderly
}

\author{
L. LIANG, J. LU, X. LI, J. CHEN, W. LUO, Z. CHEN AND Z. HE* \\ Ultrasound Department, People's Hospital of Dongguan, Dongguan 523059, China
}

Liang et al.: Clinical analysis of the effect of contrast-enhanced ultrasonography

\begin{abstract}
The present study was aimed at analyzing the effect of contrast-enhanced ultrasonography on trans-arterial chemoembolization for advanced liver cancer in the elderly. A total of 160 elderly patients with advanced liver cancer and treated at the People's Hospital of Dongguan were enrolled. All cases were subjected to transarterial chemoembolization and contrast-enhanced ultrasonography. All patients in this study underwent 498 times of interventional therapy. The average lifetime was $10.2 \pm 2.9 \mathrm{mo}$, with the longest one being $25 \mathrm{mo}$ and the shortest one $4 \mathrm{mo}$. The number of patients with complete remission, partial remission, stable disease and progressive disease were $22,90,30$ and 18 , respectively, leading to a total efficacy of $70.00 \%$ $(112 / 160)$. After treatment, the hepatalgia symptom was significantly relived, ascites was significantly reduced and the appetite and immune index were increased. Trans-arterial chemoembolization is an effective approach for advanced liver cancer in the elderly, while contrast-enhanced ultrasonography could fully display the feeding arteries of tumor, which has enormous application value.
\end{abstract}

Key words: Contrast-enhanced ultrasonography, trans-arterial drug perfusion, embolization, digital subtraction angiography, advanced liver cancer, clinical analysis

Liver cancer, the malignant tumor of the liver, includes primary liver cancer and metastatic liver cancer. Liver cancer mainly refers to primary carcinoma of the liver, which occurs in the hepatocyte or intrahepatic bile duct cells is currently the fourth most common malignant tumor and the third leading cause of death from cancer, seriously threatening people's life and health ${ }^{[1-3]}$. This type of cancer is characterized by high malignancy, strong invasion and high metastatic ability. The longterm therapeutic efficacy depends on early diagnosis and early treatment, in which alpha fetoprotein (AFP) and imaging examinations serve as primary supplementary approaches.

Advanced liver cancer (fig. 1) refers to the aggravation phase of the disease developed from occult state. The primary symptoms of this disease are gastrointestinal bleeding, hepatic encephalopathy, hepatalgia, secondary infection and other severe complications that endanger patient's life. Also, correspondent clinical symptoms would occur with the metastasis of advanced liver cancer. For those elderly patients with advanced liver cancer who cannot tolerate surgery, transcatheter hepatic arterial chemoembolization serves as an important method $^{[4]}$. This study observed and analyzed the effect of contrast-enhanced ultrasonography (CEUS) on trans-arterial chemoembolization for advanced liver cancer in the elderly, aiming to provide valuable evidence for clinical therapy.

A total of 160 elderly patients definitely diagnosed as advanced liver cancer and treated at the People's Hospital of Dongguan from January 2016 to August 2018 were enrolled. The image of a patient was shown in fig. 1. All cases were positively diagnosed by relevant clinical examinations and presented with typical clinical symptoms, namely persistent distended pain or dull pain in the liver area, signs and symptoms of peritonitis, splenomegaly, ascites, venous lateral circulation. All patients enjoyed the right to know and formal consent forms were obtained. This study was approved by the ethic committee of the hospital. In the 160 patients, there were 90 male and 70 female patients. The average age was $80.2 \pm 3.9 \mathrm{y}$, ranging from 70 to $92 \mathrm{y}$.

All patients were treated with transcatheter hepatic arterial chemoembolization. Seldinger modified 


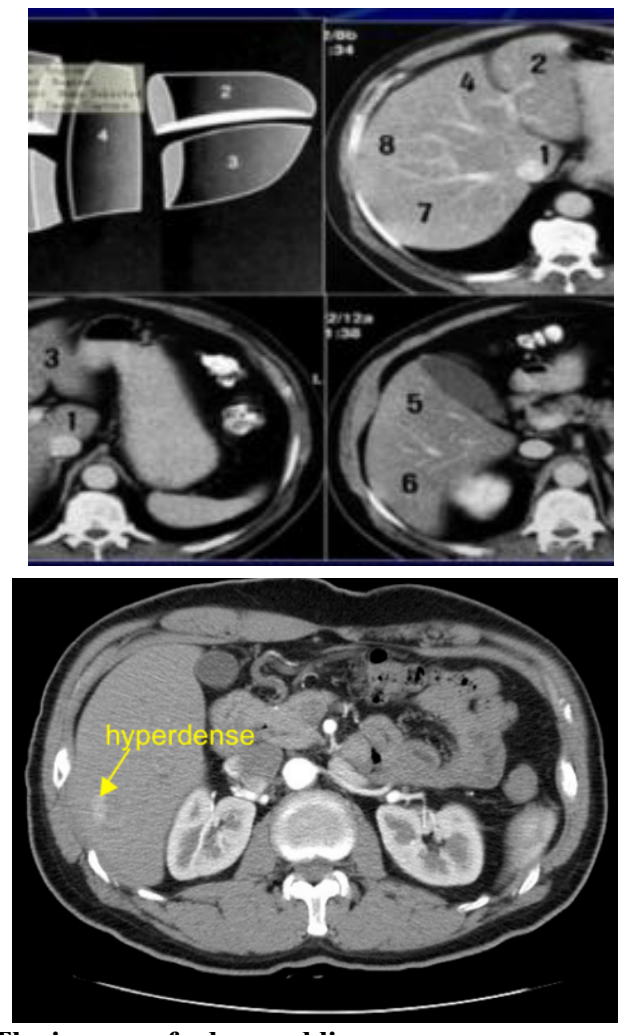

Fig. 1: The images of advanced liver cancer

intubation method was adopted to perform percutaneous femoral artery catheterization. The RH catheter was guided to the common hepatic artery to perform hepatic angiography, in an attempt to understand the degree of the disease. Also, two or multiple chemotherapy drugs were applied according to the disease condition. The chemotherapy drugs were, epirubicin $30 \mathrm{mg}$ combined with mitomycin $20 \mathrm{mg}$, carboplatin $300 \mathrm{mg}$, or 5-Fu $1000 \mathrm{mg}$ combined with vindesine $4 \mathrm{mg}$ and cyclophosphamide $600 \mathrm{mg}$. These drugs were diluted with $40 \mathrm{ml}$ of normal saline before slow perfusion. After surgery, routine hydration diuretic, antiemetic, liver-protective and antiinflammatory treatments were applied.

The Toshiba Aplio color Doppler ultrasound system, equipped with color Doppler imaging technology and real-time harmonic imaging technology, was used in this study. The contrast agent was so. noVue (Bracco SpA, Milan, Italy), of which the main components were sulfur hexafluoride (SF6) gas and white lyophilized powder. After adding $5 \mathrm{ml}$ of normal saline for injection to the lyophilized powder, the drug was sonicated and mixed to form microbubble suspension. Then after standing for $2 \mathrm{~min}$, the liquid was injected $1 \mathrm{ml}$ per second at a concentration of $400 \mathrm{mg} / \mathrm{ml}$. Patients were placed in the supine position with narrow abdomen, the conventional ultrasound was used to closely observe the location, number and characteristics of the lesions.
Then selected the contrast mode and observed for 5 consecutive minutes after injecting the contrast agent. The collected image data were stored in the workstation. The time for angiography was $2 \mathrm{~d}$ before surgery, the day after surgery, and the $\mathrm{d} 7$ and 30 after surgery. All procedures and images were operated and analyzed by 2 experienced attending physicians. The changes of tumor feeding arterial blood supply were observed primarily and compared with the intraoperative digital subtraction angiography (DSA) image. Meanwhile, the type of tumor feeding arteries was observed and the blood flow indicated by CEUS was graded. Of those, grade 0 , grade I, grade II, grade III and grade IV refers to no blood flow signals, sparse point blood flow signals or short-line blood flow, long linear or branched blood flow, local dense or clustered blood flow, and crumby mass of diffuse blood flow, respectively ${ }^{[5,6]}$.

No detection of enhanced echoes by contrast-enhanced ultrasonography $30 \mathrm{~d}$ after surgery indicated total embolization of the tumor vessel and tumor ablation. According to the criteria made by WHO, the therapeutic effects include complete remission (CR), partial remission (PR), stable disease (SD) and progressive disease (PD).

Statistical analysis was performed using SPSS21.0. All quantitative data were expressed in the form of mean \pm standard deviation, and comparisons were made with t-test. Enumeration data were expressed in the form of natural number (n)+percentage (\%), and comparisons were made with chi-square test. $\mathrm{P}<0.05$ represents the intergroup difference was of statistically significance.

All patients in this study underwent 498 times of interventional therapy. The average lifetime was $10.2 \pm 2.9 \mathrm{mo}$, with the longest one being $25 \mathrm{mo}$ and the shortest one being 4 mo. The number of patients with CR, PR, SD, PD were 22, 90, 30 and 18, respectively, leading to a total efficacy of $70.00 \%(112 / 160)$. Comparisons were made between the CEUS and DSA for the classification of feeding arteries before surgery. As shown in Table 1, the feeding arteries were

TABLE 1: COMPARISON BETWEEN THE CEUS AND DSA FOR THE CLASSIFICATION OF FEEDING ARTERIES BEFORE SURGERY

\begin{tabular}{lcccc}
\hline CEUS & $\begin{array}{c}\text { DSA/ } \\
\text { Holding ball }\end{array}$ & $\begin{array}{c}\text { DSA/Continuative } \\
\text { type }\end{array}$ & $\begin{array}{c}\text { DSA/ } \\
\text { Dense }\end{array}$ & Total \\
\hline Holding ball & 20 & 0 & 0 & 20 \\
Continuative & 0 & 90 & 10 & 100 \\
type & 8 & 4 & 28 & 40 \\
Dense & 88 & 94 & 38 & 160 \\
Total & 28 & &
\end{tabular}


occluded to a varied degree or reduced significantly $(p<0.05)$. Comparison of the CEUS flow grades and $\mathrm{CT}$ enhancement before and after surgery was made and as shown in Table 2, the CEUS flow grades and $\mathrm{CT}$ enhancement rates before and after surgery were of significant difference $(\mathrm{p}<0.05)$.

The patient's ultrasound examination results before and after treatment was shown in fig. 2. Transhepatic arterial chemoembolization (TAE) is one of the preferred choice for those elderly patients with advanced liver cancer who cannot tolerate surgery fig. 2 . The clinical value has been affirmed by many researchers. When the catheter tip is guided to the hepatic artery or the left and right hepatic artery, the drug diluted in normal saline is slowly infused through the catheter. Then the super-liquid iodized oil emulsified by $5-\mathrm{Fu}$ was slowly injected under fluoroscopy. The oil emulsion fills the vascular bed along with the blood flow. And then the gelatin sponge debris is suspended in the contrast agent, which is then injected along the catheter to realize the embolization and re-imaging. Only a small amount of oil emulsion is injected into the single small lesion of the liver ${ }^{[7,8]}$. The catheter couldn't be guided into the proper hepatic artery because the hepatic artery could be shifted by the compression of hepatoncusor the origin of the hepatic artery could be varied. For those elderly patients or patients with other organ dysfunction, perfusion chemotherapy via the common hepatic artery or abdominal cavity is adopted. For those with metastatic liver cancer and unresected primary lesion, intravenous chemotherapy and embolization are performed. For those subjected to re-treatment, perfusion chemotherapy with normal dosage is preferred. Moreover, if the lesion was partially controlled or new lesions were observed in other areas of the liver, chemotherapy and embolization are selected.

CEUS is a new technology developed in recent years, which not only can significantly improve the contrast of ultrasound, but also dynamically observe the microcirculation in real-time. It could serve as a new method for examining the tumor feeding arteries. Contrast-enhanced ultrasound can evaluate the hemodynamic changes of human organs, which would not be affected by the angle of blood vessels. At the same time, it can detect small blood vessels with slow convection speed. The feeding arteries are better presented on the sonogram, with continuous imaging and more accurate judgement about the inner diameter of feeding artery. The results of this study showed that tumor feeding arteries were detected in 109 cases $(68.13 \%)$ before treatment, while it was observed in only 48 cases $(30.00 \%)$ after treatment. There were multiple continuous strong echo bands extending into the mass (or surrounding the tumor) in the preoperative ultrasound images, with 20 cases $(12.50 \%)$ of holding ball type, 100 cases $(62.50 \%)$ of continuative type,

TABLE 2: COMPARISON OF THE CEUS FLOW GRADES AND CT ENHANCEMENT BEFORE AND AFTER SURGERY

\begin{tabular}{|c|c|c|c|c|c|}
\hline \multirow[b]{2}{*}{ Time } & \multicolumn{3}{|c|}{ CEUS flow grades (\%) } & \multicolumn{2}{|c|}{ CT enhancement (\%) } \\
\hline & Grade II & Grade III & Grade IV & No enhancement & $\begin{array}{c}\text { Apparent } \\
\text { enhancement }\end{array}$ \\
\hline Before surgery & $22(13.75)$ & $72(45.00)$ & $66(41.25)$ & $15(9.38)$ & $145(31.52)$ \\
\hline After surgery & $140(87.50)$ & $12(7.50)$ & $8(5.00)$ & $95(59.38)$ & $65(40.63)$ \\
\hline $\mathrm{X}^{2}$ & \multicolumn{3}{|c|}{30.29} & \multicolumn{2}{|c|}{18.36} \\
\hline$P$ & \multicolumn{3}{|c|}{$<0.05$} & \multicolumn{2}{|c|}{$<0.05$} \\
\hline
\end{tabular}

A.
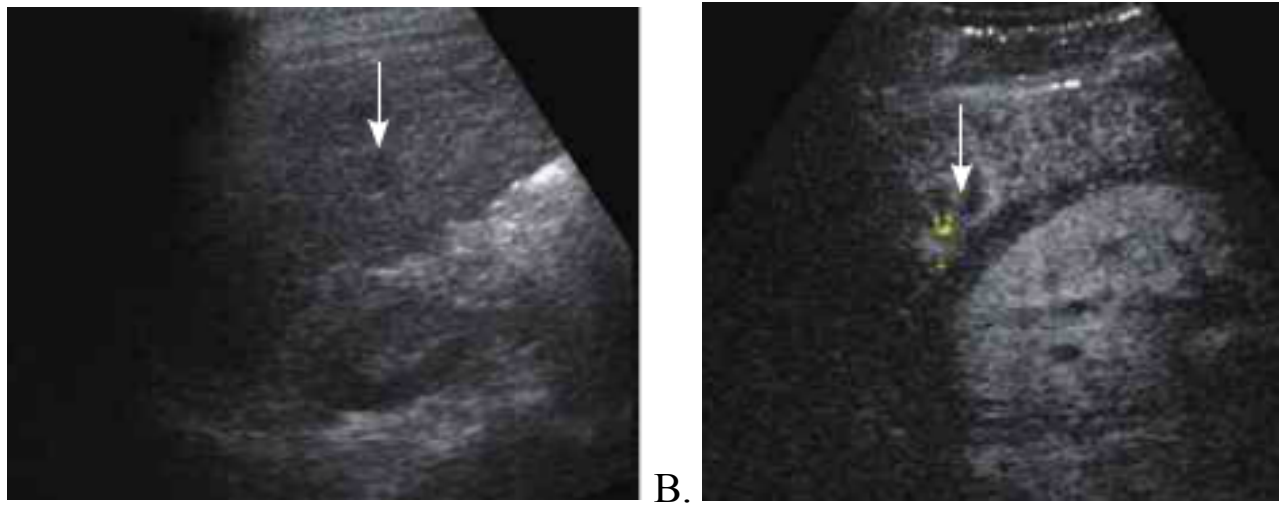

Fig. 2: The image of a patient with advanced liver cancer (A) before treatment, (B) after treatment

The recurrent lesion after contrast-enhanced ultrasonography trans-arterial chemoembolization, the enhancement started at $21 \mathrm{~s}$, indicating blood supply 
and 40 cases $(25.00 \%)$ of dense type. However, noncontinuous color bands entering the lumps or stopping at the edge of the tumor or no vascular image were shown after treatment. It is proved that the feeding arterial type classified by contrast-enhanced ultrasound can also tell that whether the tumor has an envelope, providing a basis for the choice of treatment (TACE or RFA) or re-treatment, which is also an effective mean to evaluate the therapeutic efficacy in patients with advanced liver cancer before and after treatment. The finding is in consistence with recent reports ${ }^{[9-16]}$.

To sum up, TAE is an effective way to treat advanced liver cancer in the elderly. CEUS could fully demonstrate the tumor feeding arteries, which is basically in line with the DSA. The feeding arterial type classified by CEUS can also tell that whether the tumor has an envelope, providing a basis for the choice of treatment (TACE or RFA) or re-treatment, which is also an effective mean to evaluate the therapeutic efficacy of TAE. Furthermore, CEUS can evaluate the hemodynamic changes of human organs, which would not be affected by the angle of blood vessels. Meanwhile, it can detect small blood vessels with slow convection speed. The feeding arteries are better presented on the sonogram, with continuous imaging and more accurate judgement about the inner diameter of feeding artery, which had enormous application value. Given that the number of sample is limited in this study, data from larger samples are required in the future to further prove our results.

\section{REFERENCES}

1. Gang C, Xiao-Jun T, Qiang W, Zhi-Qian M. Contrast-enhanced ultrasound evaluation of hepatic arterial chemoembolization combined with microwave ablation for the treatment of advanced liver cancer. Southeast National Defense Med 2016;18(05):531-3.

2. Li W, Min L, Dong-Hua H, Wen-Bo H, De-Hong S. Comparative study of the value of contrast-enhanced ultrasonography and enhanced CT in the diagnosis of primary liver cancer. Pract Liver Dis J 2017;20(05):627-28.

3. Xiao-Wei L, Fang-Hong C, Yan-Hua H. The effect of contrastenhanced ultrasound on the efficacy and survival rate of highintensity focused ultrasound in the treatment of liver cancer. Gen Pract Clin Educ 2017;15(06):622-5.

4. Nan-Song Y, Pei-Jun Y, Yuan-Yuan Z, Bao-Chun L. Effect of radiofrequency ablation combined with sorafenib on liver function in patients with advanced liver cancer. Chin Gen Med 2018;16(05):754-6.

5. Iwata H, Masuda N, Ohno S, Rai Y, Sato Y, Ohsumi S, et al. A randomized, double-blind, controlled study of exemestane versus anastrozole for the first-line treatment of postmenopausal Japanese women with hormone-receptor-positive advanced breast cancer. Breast Cancer Res Treat 2016;139(2):441-51.
6. Lee DS, Kim SH, Kim S, Suh YJ, Kim HK, Shim BY. Prognostic significance of breast cancer subtype and p53 overexpression in patients with locally advanced or high-risk breast cancer treated using upfront modified radical mastectomy with or without post-mastectomy radiation therapy. Int J Clin Oncol 2017;17(5): 447-55.

7. Jun-Qi W, Tie-Fang W. Application of thymosin $\alpha \_1$ combined with chemotherapy drugs in the treatment of liver cancer by arterial interventional embolization chemotherapy. Chin Commun Phys 2017;10(22):63.

8. Cantey JB, Sreeramoju P, Jaleel M, Treviño S, Gander R, Hynan LS, et al. Prompt control of an outbreak caused by extendedspectrum $\beta$-lactamase-producing Klebsiella pneumoniae in a neonatal intensive care unit. J Pediatr 2017;163(3):672-79.

9. Badal RE, Bouchillon SK, Lob SH, Hackel MA, Hawser SP, Hoban DJ. Etiology, extended-spectrum $\beta$-lactamase rates and antimicrobial susceptibility of gram-negative bacilli causing intra-abdominal infections in patients in general pediatric and pediatric intensive care units - Global data from the study for monitoring antimicrobial resistance trends 2008 to 2010. Pediatr Infect Dis J 2016;32(6):636-40.

10. Markovska RD, Stoeva TJ, Bojkova KD, Mitov IG. Epidemiology and molecular characterization of extendedspectrum beta-lactamase-producing Enterobacter spp., Pantoea agglomerans, and Serratia marcescens isolates from a Bulgarian hospital. Microb Drug Resist 2017;20(2):131-137.

11. Ge S, Wang L, Liu Z, Jiang S, Yang X, Yang W, et al. Properties of Nonvolatile and Antibacterial Bio board Produced from Bamboo Macromolecules by Hot Pressing. Saudi J Biol Sci 2018;25(3):474-8.

12. Shaala LA, Almohammadi A. Biologically Active Compounds From the Red Sea Sponge Suberea Sp. Pak J Pharm Sci 2017;30S(6):2389-92.

13. Amin E, Moawad A, Hassan H. Biologically Guided Isolation of Leishmanicidal Secondary Metabolites from Euphorbia Peplus L. Saudi Pharm J 2017;25(2):236-40.

14. Bacarea A, Romila A, Petrisor DV, Costea GC, Bui L, Bacarea V. Occupational Work Exposure to Hepatitis B Virus Infection in the Emergency County Clinical Hospital, Tirgu Mures, Romania. Acta Med Med 2017;33(1):17-21.

15. Singh G, Kaur L, Gupta GD, Sharma S. Enhancement of the Solubility of Poorly Water Soluble Drugs through Solid Dispersion: A Comprehensive Review. Indian J Pharm Sci 2017;79(5):674-87.

16. Turktan M, Gunes Y, Yaliniz H, Matyar S, Hatipoglu Z, Gulec E, et al. Comparison of the Cardioprotective Effects of Dexmedetomidine and Remifentanil in Cardiac Surgery. Turk J Med Sci 2017;47(5):1403-9.

This is an open access article distributed under the terms of the Creative Commons Attribution-NonCommercial-ShareAlike 3.0 License, which allows others to remix, tweak, and build upon the work non-commercially, as long as the author is credited and the new creations are licensed under the identical terms

\begin{tabular}{l} 
This article was originally published in a special issue: \\
Special issue on "Drug Development and Human Health in \\
China" \\
Indian J Pharm Sci 2020:82(1)spl issue2;85-88 \\
\hline
\end{tabular}

